\title{
Structure and Surface Passivation of Ultrathin Cesium Lead-Halide Nanoplatelets Revealed by Multilayer Diffraction
}

\author{
Stefano Toso ${ }^{1,2, *}$, Dmitry Baranov ${ }^{1, *}$, Cinzia Giannini ${ }^{3,{ }^{*}}$, Liberato Manna ${ }^{1,{ }^{*}}$ \\ ${ }^{1}$ Nanochemistry Department, Istituto Italiano di Tecnologia, Via Morego 30, 16163, Italy \\ ${ }^{2}$ International Doctoral Program in Science, Università Cattolica del Sacro Cuore, 25121 Brescia, Italy \\ ${ }^{3}$ Istituto di Cristallografia - Consiglio Nazionale delle Ricerche (IC-CNR), Via Amendola 122/O, I-70126 Bari, Italy \\ *Corresponding authors: stefano.toso@iit.it, dmitry.baranov@iit.it, cinzia.giannini@ic.cnr.it, liberato.manna@iit.it
}

Keywords: multilayer diffraction, nanoplatelet, perovskite, Ruddlesden-Popper, lead halide, X-ray, surface, structure

\begin{abstract}
The research on bidimensional colloidal semiconductors has received a boost from the emergence of ultrathin lead-halide perovskite nanoplatelets. While the optical properties of these materials have been widely investigated, their accurate structural and compositional characterization is still challenging. Here, we exploited the natural tendency of the platelets to stack into highly ordered films, which can be treated as single crystals made of alternating layers of organic ligands and inorganic nanoplatelets, to investigate their structure by Multilayer Diffraction. Using X-ray diffraction alone, this method allowed to refine the structure of $\sim 12 \AA$ thick Cs-Pb-Br perovskite and $\sim 25 \AA$ thick Cs-Pb-Cl-I Ruddlesden-Popper nanoplatelets by precisely measuring their thickness, stoichiometry, surface passivation type and coverage, as well as deviations from the crystal structures of the corresponding bulk materials. It is noteworthy that a single, readily available experimental technique, coupled with proper modeling, provides access to such detailed structural and composition information.
\end{abstract}

\section{Introduction}

Colloidal semiconductor nanoplatelets are materials characterized by large exciton binding energy and oscillator strength, sharp spectral features ${ }^{1-4}$ and short photoluminescence lifetimes, ${ }^{2}$ all properties that make them appealing in devices such as LEDs and lasers. ${ }^{2,5-7}$ The extreme thinness, down to just a few atoms, is both the source of their unique optical properties and a major challenge for their structural characterization. Several studies have demonstrated that nanoplatelets can be much different from an ideal "slice" of the corresponding bulk material: they are structurally less constrained than the bulk and can readily relax through structural distortions and altered lattice constants. ${ }^{8}$ For example, the rock-salt cubic PbS becomes orthorhombic when shaped into thin platelets, ${ }^{9}$ and $\mathrm{CdSe}$ zincblende nanoplatelets are better described by a tetragonal structure rather than the cubic structure of the bulk. ${ }^{10}$ Furthermore, due to their high surface-to-volume ratio the surface layers become a relevant fraction of the whole nanoplatelet, to the point that the type of surface termination affects the overall stoichiometry of the platelets and their physical properties. $^{2,11}$

The emergence of colloidal lead-halide perovskites considerably expanded the interest in nanoplatelets in the past five years. Lead-halide perovskite nanoplatelets can be synthesized with excellent 
control over their thickness from six down to one single $\left[\mathrm{PbX}_{6}\right]^{4-}$ octahedron, providing access to a level of quantum confinement that would be challenging to achieve in isotropic nanocrystals. ${ }^{1,2,8,12}$ However, significantly less is known about these particles if compared with their cuboidal nanocrystal counterparts. Because of the intrinsic structural softness of halide-perovskites and the symmetry breaking due to their finite thickness, ${ }^{13-16}$ such platelets may undergo a structural reorganization if compared to the bulk, which is challenging to capture in its fine details. Moreover, as anticipated above the extreme thinness of platelets makes their surface a considerable fraction of their entire volume, therefore requiring a careful investigation of their termination layers. Finally, films of lead halide perovskite nanoplatelets are actively explored for use in light-emitting devices. In such films the platelet orientation, the interplatelet distance, and the stacking disorder affect the electronic coupling between nanoplatelets, their dielectric screening, and the orientation of their transition dipole moments. Therefore, such parameters can be relevant for tuning the optical characteristics of the device. ${ }^{17-19}$

To retrieve all this information, a combination of several techniques is typically required. For example, high-resolution transmission electron microscopy (HR-TEM) is commonly used to investigate the structure of nanoplatelets. ${ }^{20}$ These, however, often lie flat with respect to the electron beam, and TEM is blind to their more elusive thin dimension unless in perfectly vertical stacks, a rarely met condition for thin and laterally extended platelets. ${ }^{21}$ Furthermore, curling and bending can prevent achieving the atomic resolution in ultrathin nanoplatelets, and the electron beam can cause severe damages. ${ }^{8,10,22} \mathrm{X}$-ray powder diffraction is a less invasive, more versatile and sensitive technique than TEM, but diffraction signals from nanoplatelets are smeared due to their limited periodicity along the thin direction. Thus, Bragg diffraction and its derived methods such as Rietveld refinement are poorly suited to characterize nanoplatelets, which are better studied with Total Scattering methods. ${ }^{8,10}$ However, these methods rely on high-quality data, usually from synchrotrons, ${ }^{23}$ and require an a priori 3D atomistic model of the entire nanoparticle to start with. In addition, Total Scattering methods are usually applied on samples composed of randomly oriented crystallites: their extention to films of oriented nanoparticles requires introducing corrections for the preferred orientation, which would add considerable complexity to the method.

In this work, we investigate the structure of colloidal cesium lead-halide nanoplatelets along their thinnest dimension through the application of Multilayer Diffraction analysis to highly ordered nanoplatelet films. ${ }^{24-26}$ The single- and mixed-halide $\mathrm{Cs}-\mathrm{Pb}-\mathrm{X}(\mathrm{X}=\mathrm{Br}$ or $\mathrm{Cl}-\mathrm{I})$ nanoplatelets were prepared by colloidal synthesis and self-assembled into thin films by slow solvent evaporation. The self-assembly process was exploited to create an extended periodicity along the thin direction of the nanoplatelets, producing a peculiar pattern of periodic peaks when the film is subject to a $\theta: 2 \theta$ diffraction experiment. By applying the knowledge derived from the earlier work on lead-halide nanocrystal superlattices, ${ }^{24,25}$ we developed an algorithm that enables the refinement of the nanoplatelets structure by means of a full-profile multiparametric fitting of their diffractogram. Despite relying only on a widely available lab-grade diffractometer, the amount of structural and compositional information that can be retrieved is noteworthy. When the method was applied to $\mathrm{Cs}-\mathrm{Pb}-\mathrm{Br}$ nanoplatelets, it demonstrated that they were two-monolayer (i.e., two $\left[\mathrm{PbBr}_{6}\right]^{4-}$ octahedra) thick, stacked at about $\sim 34 \AA$ from each other with a standard deviation of only $\sim 0.5 \AA$. Furthermore, the method enabled us to discern the surface termination of the platelets, that consisted of a partially defective plane of $\mathrm{Br}^{-}$ions and $\mathrm{NH}_{3}-\mathrm{R}^{+}$ligands, and to demonstrate that oleic acid, although present during the synthesis, played no role in their surface passivation. We also measured the anisotropic structural expansion of nanoplatelets with respect to bulk $\mathrm{CsPbBr}_{3}$, confirming a behavior reported for cesium lead-halide nanostructures in the past. . $^{84,27}$ 
While a relatively simple structure and a well-documented relationship between thickness and optical properties eases the identification of the number lead-halide octahedra layers in the case of $\mathrm{Cs}-\mathrm{Pb}-\mathrm{Br}$ nanoplatelets, the presented method enables the investigation of more elusive materials as well. Here we report the example of $\mathrm{Cs}_{2} \mathrm{PbCl}_{2} \mathrm{I}_{2}$ Ruddlesden-Popper nanoplatelets, for which it is not possible to determine the number of layers from optical spectra. Nonetheless, for this system the Multilayer Diffraction approach enabled us to accurately measure a thickness of three $\left[\mathrm{PbCl}_{4} \mathrm{I}_{2}\right]^{4-}$ octahedra, capture the anisotropic expansion of the structure along its thin dimension, and determine the nature of the surface termination layer, that is composed of $\mathrm{I}^{-}$and $\mathrm{NH}_{3}-\mathrm{R}^{+}$ions. Contrary to our initial expectations, we also found that the $\mathrm{Cs}^{+}$ and $\mathrm{I}^{-}$ions form a common atomic plane in nanoplatelets, as opposed to being located in separate planes as in bulk $\mathrm{Cs}_{2} \mathrm{PbCl}_{2} \mathrm{I}_{2}$, providing yet another example of structural differences between a nanoparticle and the corresponding bulk material.

\section{Results}

\section{Sample preparation, data processing and model outline}

In this work, we prepared $\mathrm{Cs}-\mathrm{Pb}-\mathrm{Br}$ nanoplatelets with a lateral dimension of a few dozens of nanometers (Figure 1a) via a previously published recipe. ${ }^{28}$ The films of oriented nanoplatelets for the Multilayer Diffraction analysis were prepared by slowly drying a suspension of nanoplatelets in hexane on top of a tilted silicon wafer. Upon the successful film deposition, a region of the substrate became covered by a smooth and iridescent film, and was selected for the diffraction experiment. As a matter of fact, a brilliant and homogeneous color over extended areas, originated by thin-film light interference, attests to the expected constant thickness and limited roughness of the film (Figure 1b). ${ }^{29}$ The samples were probed in $\theta: 2 \theta$ out-of-plane diffraction experiments, producing diffraction signals in the form of equally spaced fringes whose intensity and sharpness decreased at higher angles. Such diffraction fringes appear when the structural perfection of the multilayer reaches the Angstrom level, ${ }^{24}$ far beyond what can be probed optically, and encode the structure of the film along its vertical direction. This includes the overall periodicity of the multilayer and its stacking disorder as well as the vertical coordinates and the electron densities of all the atomic planes in the sample.

However, to extract this information we must refine a starting model of the multilayer by minimizing the differences between the experimental data and a simulated diffractogram, similarly to what is done in Rietveld refinement. First, the data must be prepared for the analysis by subtracting the instrumental background together with any residual reflection from misaligned nanoplatelets or from the substrate. In addition, the Lorentz-Polarization-Absorption correction must be applied to compensate for the instrumental contributions to the measured intensity (LPA, Figure $1 \mathrm{c}$ ). ${ }^{26}$ This step is needed to make the experimental pattern comparable with the simulation. In fact, the so-processed data are an experimental measurement of $\left|F_{M L}(q)\right|^{2}$, that is the square modulus of the multilayer structure factor $F_{M L}(q)$ describing the amplitude and phase of X-rays diffracted by the multilayer. Our analysis relies on the fact that $F_{M L}(q)$ can be simulated starting from a structural representation of the multilayer.

To achieve such description, the multilayer can be broken down into two parts: the high-density inorganic nanoplatelets and the low-density interparticle spacings composed of organic ligands. The periodic alternation of low- and high-density layers is responsible for the strong electron density contrast in the sample, which in turn produces the multilayer diffraction fringes. Due to their low density, the interparticle spacings minimally contribute to the signal intensities across the experimentally studied q-range, and for the 
purpose of the analysis they can be approximated as amorphous carbon layers with fixed density. The most important part of the multilayer are the nanoplatelets, whose structure factor $F_{N P}(q)$ is the main component of $F_{M L}(q)$. In this work, we describe $F_{N P}(q)$ in its most essential form, that is the sum in phase of the radiation scattered by each individual atom $j: 30$

$$
F_{N P}(q)=\sum_{j} f_{j}(q) \cdot e^{-i \vec{q} \cdot \overrightarrow{R_{j}}} \quad \text { Eq. } 1
$$

Where $f_{j}$ is the element-specific atomic form factor, $\vec{q}$ is the scattering vector, and $\overrightarrow{R_{j}}=\hat{x} \cdot x_{j}+\hat{y} \cdot y_{j}+\hat{z} \cdot$ $z_{j}$ is the position vector of the $j^{\text {th }}$ atom inside the nanoplatelet. Computing the structure factor in this way would be demanding for platelets containing dozens of thousands of atoms; however, developing Equation 1 one step further immediately highlights the huge advantage of dealing with ordered multilayers instead of randomly oriented nanoplatelets. During a $\theta: 2 \theta$ out-of-plane diffraction experiment the scattering vector $\vec{q}$ is perpendicular to the sample surface plane $x y$, therefore $\vec{q} \cdot \overrightarrow{R_{J}}=q \cdot z_{j}$ :

$$
F_{N P}(q)=\sum_{j} f_{j}(q) \cdot e^{-i q \cdot z_{j}} \quad \text { Eq. } 2
$$

As small as it might seem, this change has a major impact on the data treatment. Instead of considering a complex 3D model (Figure 1d), we can describe the structure entirely by the handful of atoms needed to capture the composition of each atomic plane along the $z$ direction. For example, two $\left[\mathrm{PbBr}_{6}\right]^{4-}$ octahedra thick $\mathrm{Cs}-\mathrm{Pb}-\mathrm{Br}$ nanoplatelets are described by just 10 atoms divided in 5 layers: $\mathrm{Br}-\mathrm{PbBr}_{2}-\mathrm{CsBr}-\mathrm{PbBr}_{2}-\mathrm{Br}$ (Figure 1e). Based on this representation, our algorithm computes $F_{N P}(q)$ and then exploits it to calculate $F_{S L}(q)$ by introducing the effects of the multilayer periodicity and stacking disorder. Finally, $F_{S L}(q)$ is turned into $\left|F_{M L}(q)\right|^{2}$, producing a simulation that can be matched with the experimental data (Figure $\left.1 \mathrm{f}\right)$. Additional details on the sample preparation, the data analysis and the diffractogram simulation can be found in the Methods section and in the SI, section S1-3.

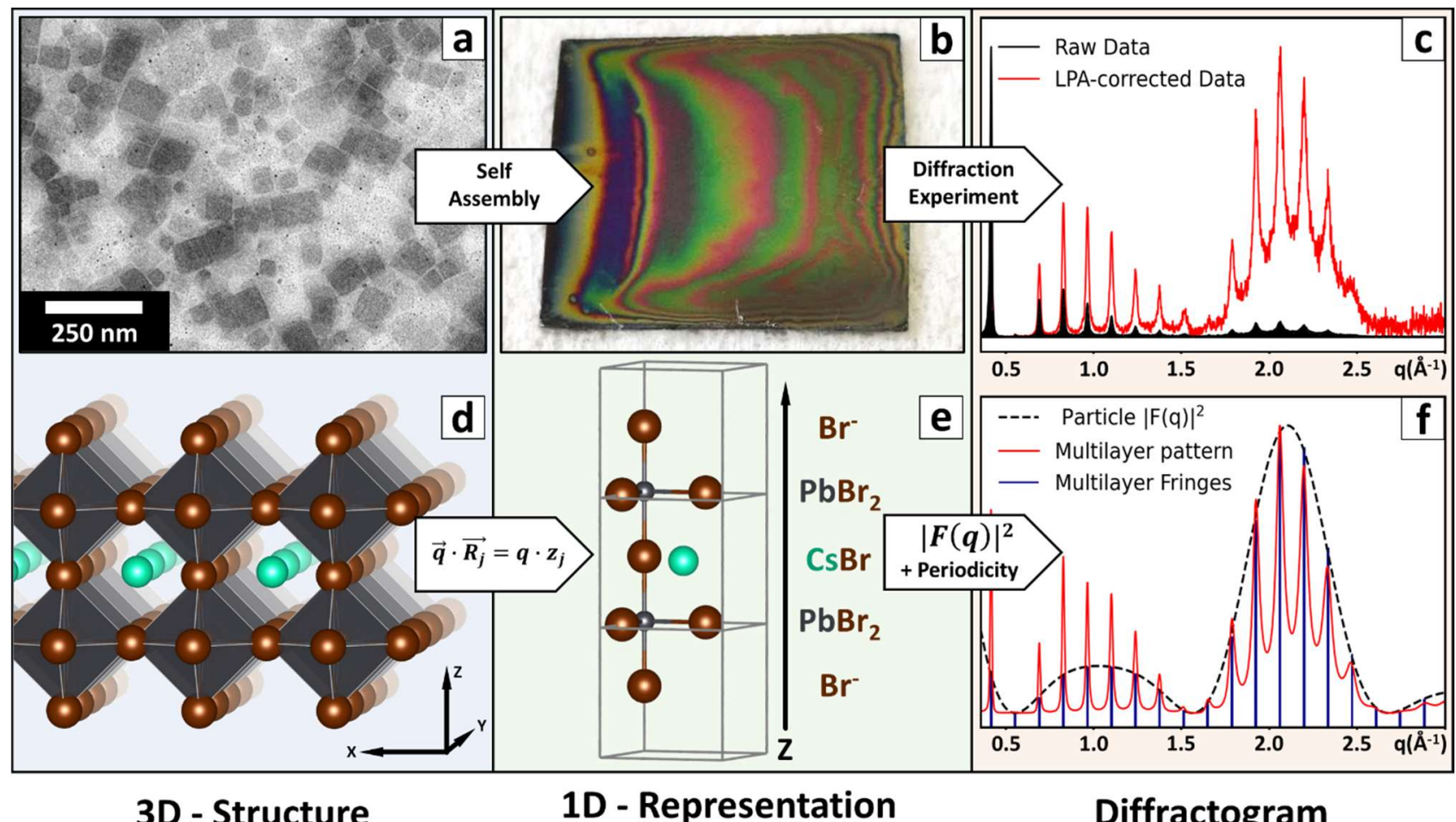

Figure 1. Experimental and simulated Multilayer Diffraction patterns. Slow solvent evaporation drives selfassembly of (a) colloidal nanoplatelets of uniform thickness into (b) highly oriented iridescent thin films on 
top of a silicon wafer. In such films, the electron-dense platelet and the comparatively electron-light ligands alternate along the vertical direction. These are multilayered systems, that modulate the intensity of diffracted X-rays yielding patterns characterized by periodic sharp fringes. Such experimental patterns (c, black solid line "Raw Data") can be conveniently exploited to refine the structure of the nanoplatelets by comparing them with a simulation once the Lorentz-Polarization-Absorption correction has been applied (c, red solid line "LPA-corrected Data"). In fact, the ordered stacking into a multilayer allows to reduce the 3D structure of randomly oriented nanoplatelets (d) to a 1D representation of the film along its z-axis (e). Such representation is used to compute the nanoplatelet structure factor $F_{N P}(q)$ through the Equation 2, and then to convolute its square modulus $\left|F_{N P}(q)\right|^{2}$ (f, black dashed line) with the fringes arising from the nanometerscale periodicity of the multilayer ( $f$, vertical blue lines). This produces a simulation of the whole multilayer diffractogram ( $f$, red solid line), that can be matched with the experimental data to refine the input 1D representation of the nanoplatelet structure.

\section{Structural refinement of $\mathrm{Cs}-\mathrm{Pb}-\mathrm{Br}$ nanoplatelets}

The model we outlined allows to refine the structure of $\mathrm{Cs}-\mathrm{Pb}-\mathrm{Br}$ nanoplatelets via the optimization of a consistent starting model, that is a 1D representation of the nanoplatelets where the number of atomic planes, their stacking sequence, and the elements they contain is imposed a priori. For quantum-confined platelets the thickness can be estimated through the absorption spectrum: in our case, it matched with reports for $\sim 12 \AA$ thick nanoplatelets (excitonic absorption peak at $428 \mathrm{~nm}$, Figure 2a). ${ }^{12,28,31}$ Taking the $\mathrm{CsPbBr} 3$ crystal structure as a reference, where the $\mathrm{Pb}-\mathrm{Br}$ bonds are about $\sim 3 \AA$ long, the nanoplatelets must be composed of 5 alternating $\mathrm{CsBr}$ and $\mathrm{PbBr}_{2}$ layers. To complete the nanoplateled model, we had to provide a description for the the surface as well. In fact, as a consequence of the extreme thinness the surface layers are a significant fraction the entire nanoplatelet, as they constitute 2 out of 5 atomic layers along the thin direction. Such a high contribution is expected to leave a clear mark on the diffraction pattern, therefore giving insight into the nature of the surface passivation layer. To elucidate this aspect, we considered three surface models that have been previously proposed for $\mathrm{CsPbBr}_{3}$ nanocrystals: $\mathrm{PbBr}_{2}$-termination, $\mathrm{CsBr}$ termination and $\mathrm{Br}^{-} / \mathrm{R}^{-} \mathrm{NH}_{4}{ }^{+}$termination (Figure $2 \mathrm{~b}-\mathrm{d}$, insets). ${ }^{32-36} \mathrm{~A}$ preliminary comparison between calculated and experimental patterns performed by refining only few selected parameters ( $\mathrm{Pb}-\mathrm{Pb}$ distance, interparticle spacing, stacking disorder), easily discarded the $\mathrm{PbBr}_{2}$ - and $\mathrm{CsBr}$-terminations (Figure 2b-c), while the $\mathrm{Br} / \mathrm{R}_{-}-\mathrm{NH}_{4}{ }^{+}$termination produced a good match with the experimental data (SI, Section S4).

The match between the measured and calculated diffractograms could be further improved by refining the fractional occupancies of the atoms in each plane, that is described in the model by multiplying each atomic form factor $f_{j}$ in Equation 2 by a number in between 0 (zero occupancy) and 1 (full occupancy). The resulting picture of the nanoplatelets structure is hereby presented (Figure $2 \mathrm{~d}$, see SI, Section S5 for further details on the analysis). The $\mathrm{Cs}-\mathrm{Pb}-\mathrm{Br}$ nanoplatelets were confirmed to be composed of two $\left[\mathrm{PbBr}_{6}\right]^{4-}$ octahedra layers, for an overall thickness of $11.848 \pm 0.008 \AA$, and were separated by a layer of organic ligands $34.00 \pm 0.01 \AA$ thick with a stacking disorder parameter of $0.505 \pm 0.007 \AA$ (i.e. the standard deviation of the interparticle distance). This corresponds to an overall multilayer periodicity of $45.89 \pm 0.01 \AA$. The most electron-dense atomic layers, namely the $\mathrm{PbBr}_{2}$ ones, were assumed to be fully occupied to serve as a reference for refining the relative occupancies of all the other layers. With respect to this reference, the $\mathrm{CsBr}$ layer in the middle of the platelet was found to be slightly-to-non defective (occupancy $=95 \pm 1 \%$ ), while the surface $\mathrm{Br} / \mathrm{R}-\mathrm{NH}_{4}{ }^{+}$layer was significantly defective (occupancy $=73 \pm 2 \%$ ). The nanoplatelet structure was found to be expanded along the thin direction: the $\mathrm{Pb}-\mathrm{Pb}$ distance is $5.924 \pm 0.004 \AA$, longer than that 
measured for the horizontal direction by $1.56 \%$ (5.833 $\AA$, from the residual signal of non-stacked platelets, see $\mathrm{SI}$ section, $\mathrm{S} 2$ ). Furthermore, both $\mathrm{Pb}-\mathrm{Pb}$ distances are longer than those found in bulk $\mathrm{CsPBBr}_{3}$ (vertical $=+0.75 \%$ [010] bulk, horizontal $=+0.22 \%<101>$ bulk, Pnma setting, ICSD-97851). ${ }^{37}$ This finding is in line with the known tendency of lead halides to relax their structure anisotropically at the nanoscale, ${ }^{8,24}$ and gives insight about the lattice symmetry.

Lead-halide perovskites can adopt a cubic, tetragonal, or orthorhombic structure depending on if and how the $\left[\mathrm{PbX}_{6}\right]^{4-}$ octahedra are tilted. ${ }^{38,39}$ While the debate on bulk and large nanocrystals of $\mathrm{CsPbBr}_{3}$ has settled in favor of the orthorhombic polymorph, this aspect is still not entirely clarified for small nanocrystals and thin platelets. ${ }^{40-44}$ The different $\mathrm{Pb}-\mathrm{Pb}$ distances along the horizontal and vertical directions easily exclude the cubic symmetry: we tried to discern between the two options left based on the atoms in the $\mathrm{PbBr}_{2}$ planes being coplanar (tetragonal) or misaligned (orthorhombic) due to the tilting of octahedra (see $\mathrm{SI}$, Section S5 for details). To do so, we let the z-coordinate of the $\mathrm{Br}^{-}$ions in the $\mathrm{PbBr}_{2}$ planes free to relax, finding that $\left|\mathrm{z}_{\mathrm{Pb}}-\mathrm{Z}_{\mathrm{Br}}\right|=0.241 \pm 0.054 \AA$. This is compatible within error with the $\left|\mathrm{z}_{\mathrm{Pb}}-\mathrm{Z}_{\mathrm{Br}}\right|=0.294 \AA$ found in bulk $\mathrm{CsPbBr}_{3},{ }^{37}$ suggesting that the two-monolayer $\mathrm{Cs}-\mathrm{Pb}-\mathrm{Br}$ nanoplatelets adopt an orthorhombic structure, in line with a previous report on 6-octahedra thick nanoplatelets. ${ }^{8}$ See $\mathrm{SI}$, section $\mathrm{S} 5$ for further details on the analysis of $\mathrm{Cs}-\mathrm{Pb}-\mathrm{Br}$ nanoplatelets.
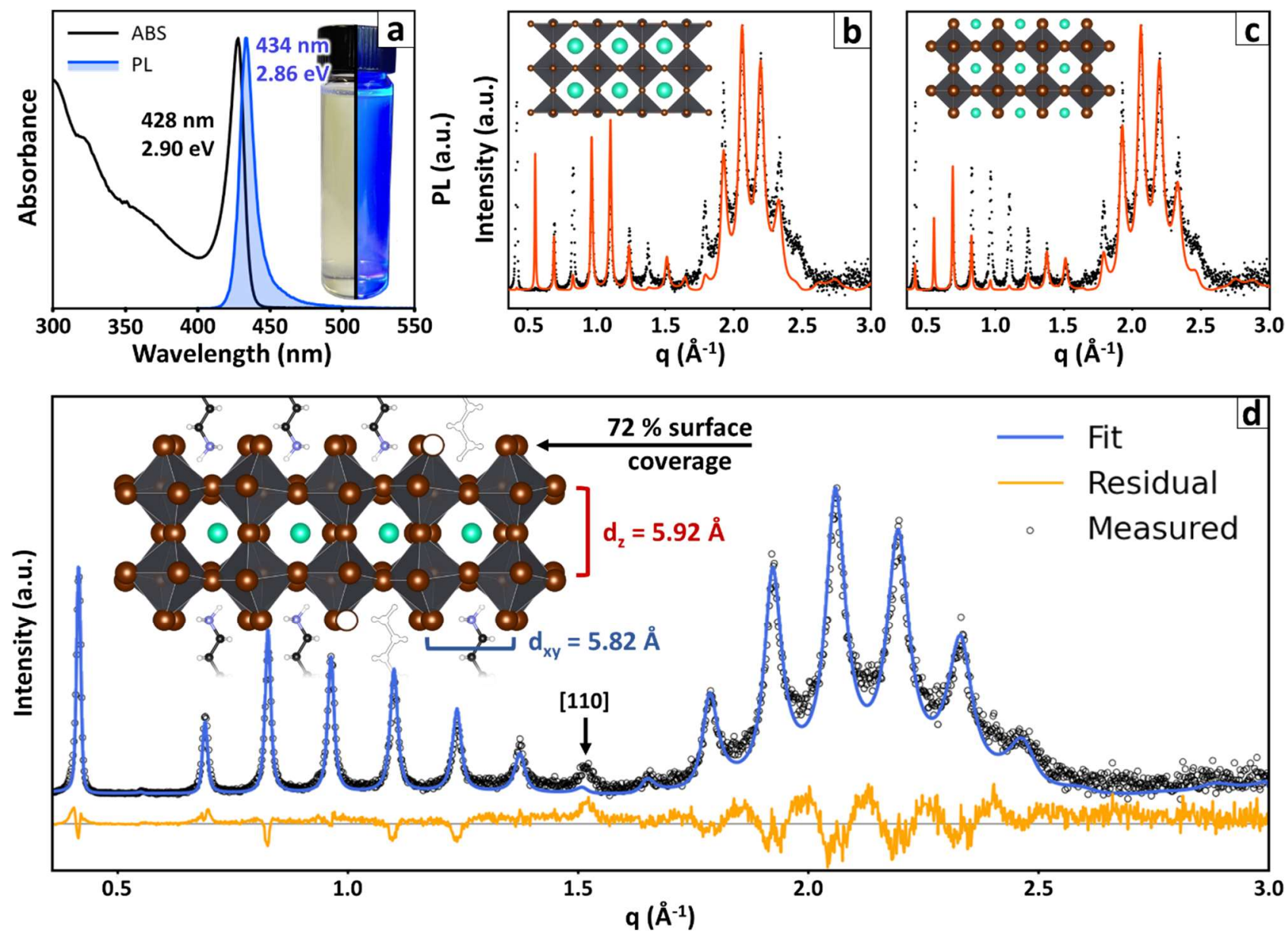

Figure 2. Structural refinement of Cs-Pb-Br nanoplatelets. (a) Absorption (ABS) and photoluminescence (PL) spectra of two $\left[\mathrm{PbBr}_{6}\right]^{4-}$ octahedra thick $\mathrm{Cs}-\mathrm{Pb}-\mathrm{Br}$ nanoplatelets. Inset: a photograph of a colloidal suspension of nanoplatelets under ambient illumination (left) and UV light (right), the latter showing the characteristic bright blue emission. Preliminary fits testing the hypotheses of (b) a $\mathrm{PbBr}_{2}$ surface termination and (c) a CsBr 
surface termination. A visual examination reveals that these surface terminations do not capture accurately the intensity of peaks in the experimental patterns. (d) Best-fit of the diffraction pattern obtained by refining the structural parameters of the $\mathrm{NH}_{4}-\mathrm{R} / \mathrm{Br}$ termination model, including the occupancies, and the vertical coordinates of atoms in the platelet structure. The residual signal at $q \sim 15 \AA^{-1}$ is not a multilayer fringe, but a peak from a fraction of misaligned platelets ([110] in the pseudocubic notation for $\mathrm{CsPbBr}_{3}$ ). The inset illustrates the structure of the nanoplatelet in scale, highlighting its expansion along the vertical direction, the partially-occupied surface layers and the tilted $\left[\mathrm{PbBr}_{6}\right]^{4-}$ octahedra. Color legend for atoms: $\mathrm{Cs}^{+}=$lightblue ; $\mathrm{Br}^{-}=$brown; $\mathrm{Pb}^{2+}=$ dark gray (octahedra); $\mathrm{N}=$ violet; $\mathrm{C}=$ black; $\mathrm{H}=$ white.

\section{On the surface passivation of $\mathrm{Cs}-\mathrm{Pb}-\mathrm{Br}$ nanoplatelets}

X-ray diffraction is not often exploited to study the surface of nanocrystals due to the predominant contribution of the volume to the overall diffracted intensity, although its weight and role is highly recognized. ${ }^{45}$ However, assembling the platelets into a multilayer effectively turned their surface into a periodic component of a larger crystal, therefore enabling its investigation. The diffraction methods are sensitive to the electron density of the sample, that depends on its chemical composition. Thus, the nanoplatelet surface experimentally appears as a layer of atoms with a lower electron density than the core, to which we must give an interpretation in terms of chemical composition. This is done by assuming a starting model passivated by a layer of $\mathrm{Br}^{-}$and $\mathrm{R}^{-} \mathrm{NH}_{4}{ }^{+}$ions, in line with prior findings and with our preliminary simulation (Figure S6a), ${ }^{32-36}$ and then refining the occupancy of surface ions by matching the electron density of the model with the one measured experimentally. For example, the $73 \%$ occupation found for the $\mathrm{Br} / \mathrm{R}$ $\mathrm{NH}_{4}{ }^{+}$surface layers indicates that they have an electron count of $\sim 31.4 \mathrm{e}^{-} /$formula unit $\left(=0.73 \cdot\left[\mathrm{e}^{-}{ }_{\mathrm{Br}}+\mathrm{e}^{-} \mathrm{N}\right]\right)$ if compared to the $\mathrm{PbBr}_{2}$ layers we took as a reference (152 $\mathrm{e} /$ formula unit). The raw data is the electron count, while the vacant layer of $\mathrm{Br} / \mathrm{R}^{-} \mathrm{NH}_{4}{ }^{+}$ions is the way our model accounted for it. In principle, many other surface terminations are compatible with the same electron count, and would be indistinguishable by relying only on the diffraction data from our experiments. Therefore, we had to validate this interpretation by independent methods.

The first hint towards a $\mathrm{Br}^{-} / \mathrm{R}^{-} \mathrm{NH}_{4}{ }^{+}$termination is the comparison with 2D-layered perovskites. Those are bulk materials where layers of $\mathrm{A}^{+}$cations of the perovskite (i.e., $\mathrm{Cs}^{+}$for $\mathrm{Cs}_{\mathrm{PbBr}}$ ) are replaced with a longchain ammonium cation, that has the effect of dissecting the perovskite structure into inorganic slices separated by the organic component. ${ }^{46,47}$ At the interface between the inorganic and the organic parts of the crystal, the polar head of the ammonium cation replaces the $A^{+}$cation. The same happens in our nanoplatelets films, where thin inorganic nanoplatelets are separated by organic molecules: it is fair to assume that the $\mathrm{Cs}^{+}$ions on the surface are replaced by the polar heads of oleylammonium ions. Based on this assumption we can calculate the theoretical elemental ratio in the sample. For vacancy-free two octahedra-thick platelets the calculated ratio comes out to be $\mathrm{Cs}: \mathrm{Pb}: \mathrm{Br}=1: 2: 7$, otherwise $0.95: 2: 6.41$ if the occupancies from the fit are included. By SEM-EDS we measured $\mathrm{Cs}: \mathrm{Pb}: \mathrm{Br}=0.93: 2: 6.43$ (see $\mathrm{SI}$, Section S6) in agreement with the proposed model.

Another open question is whether oleic acid plays a role in surface passivation of the nanoplatelets. ${ }^{33}$ Both oleylamine and oleic acid are used in the synthesis, and while an oleylammonium bromide termination easily accounts for the role of oleylamine, the presence of oleic acid cannot be easily excluded. We answered this question by characterizing self-assembled nanoplatelets that had been synthesized with either a longer carboxylic acid (erucic acid with $\mathrm{C}_{22}$ as compared to $\mathrm{C}_{18}$ in oleic aicd) or with a shorter amine (octylamine with $C_{8}$ as compared to $C_{18}$ in oleylamine), taking advantage of the sensitivity of Multilayer Diffraction to the 
interparticle spacing. The introduction of erucic acid produced no appreciable change in the diffractogram. Instead, in the octylamine-containing sample the interparticle distance dropped from $34.0 \AA$ to $15.2 \AA$ and the stacking disorder parameter decreased from $0.50 \AA$ to $0.24 \AA$. This findings confirmed the hypothesis that oleic acid plays no significant role in the passivation of the nanoplatelet surface. The results are consistent with a prior NMR study by de Roo et. al. demonstrating that oleic acid is not interacting with the surface of $\mathrm{CsPbBr} 3$ nanocrystals in liquid dispersion. ${ }^{33}$ See $\mathrm{SI}$, Section $\mathrm{S} 6$ for data and details regarding the investigation of the surface passivation.
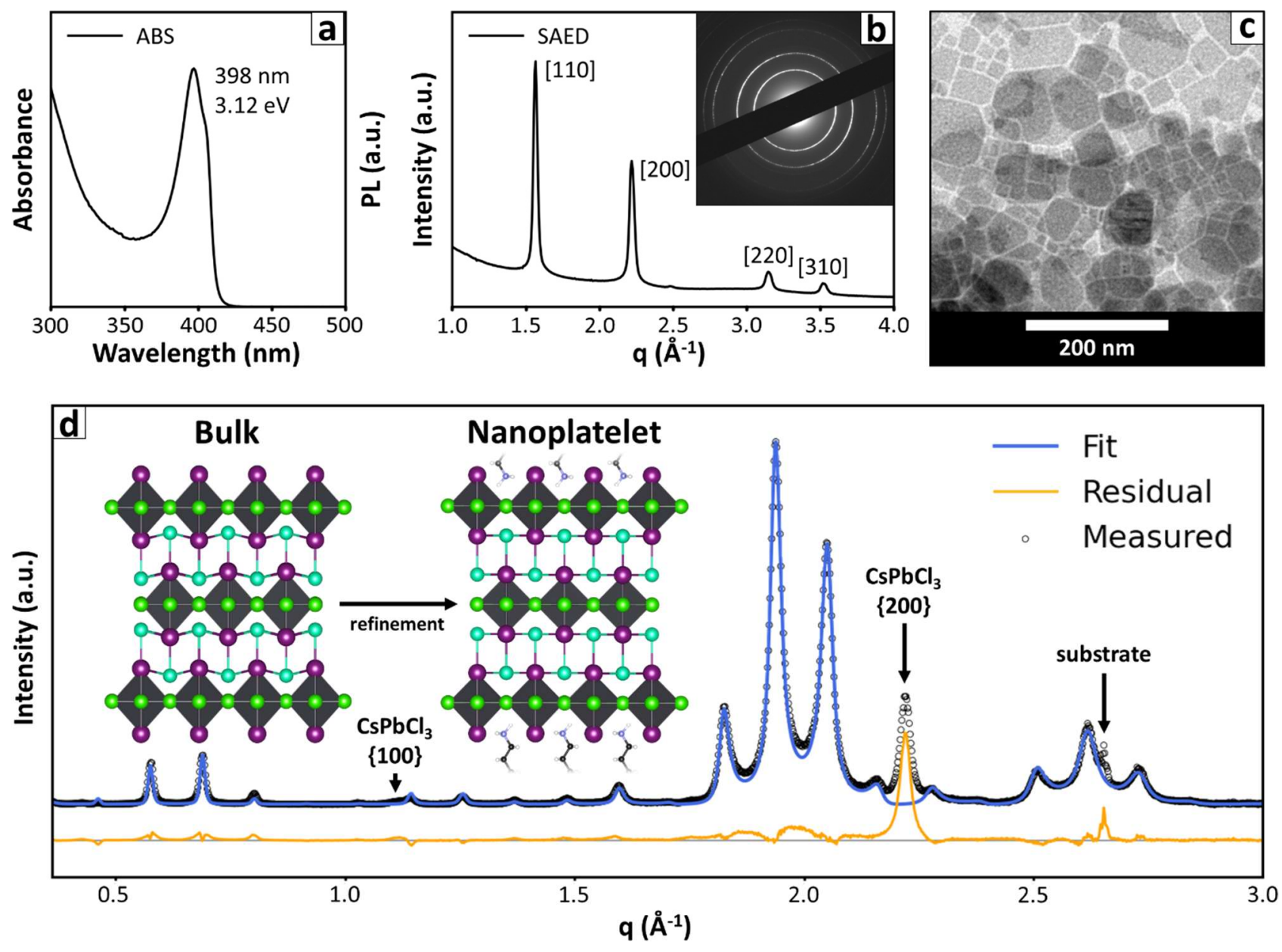

Figure 3. $\mathrm{Cs}_{2} \mathrm{PbCl}_{2} \mathrm{I}_{2}$ Ruddlesden-Popper nanoplatelets. (a) Absorption spectrum of $\mathrm{Cs}_{2} \mathrm{PbCl}_{2} \mathrm{I}_{2}$ nanoplatelets. The spectral position of the excitonic peak matches with that reported in the past for nanocrystals of the same material $(400 \mathrm{~nm} / 3.10 \mathrm{eV}),{ }^{48,49}$ and is only weakly shifted from that of bulk $\mathrm{Cs}_{2} \mathrm{PbCl}_{2} \mathrm{I}_{2}(408 \mathrm{~nm} / 3.04 \mathrm{eV})$. ${ }^{50}(\mathrm{~b}-\mathrm{c})$ TEM images and SAED diffraction pattern of $\mathrm{Cs}_{2} \mathrm{PbCl}_{2} \mathrm{I}_{2}$. The SAED pattern provides information about the structure of the platelets along the horizontal direction, and is compatible with the one expected for oriented $\mathrm{Cs}_{2} \mathrm{PbCl}_{2} \mathrm{I}_{2}$ crystals. (d) Multilayer Diffraction fit of the $\mathrm{Cs}_{2} \mathrm{PbCl}_{2} \mathrm{I}_{2}$ nanoplatelets pattern. The starting

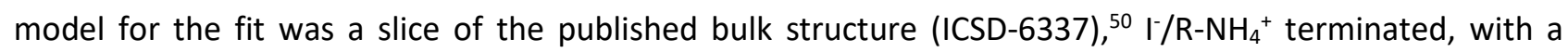
thickness of three $\left[\mathrm{PbCl}_{4} \mathrm{I}_{2}\right]^{4-}$ octahedra. In the refined structure, the $\mathrm{Cs}^{+}$and $\mathrm{I}^{-}$ions are found to converge into one common layer of ions as the platelet expands slightly along the vertical direction ( $\mathrm{Pb}-\mathrm{Pb}$ distance $+1.4 \%$ ). The residual non-fitted signals correspond to the $\{100\}$ and $\{200\}$ Bragg peaks of the $\mathrm{CsPbCl}_{3}$ impurity and to some signals from the substrate. Color legend for atoms: $\mathrm{Cs}^{+}=$light-blue; $\mathrm{Cl}^{-}=$green; $\mathrm{I}^{-}=$purple; $\mathrm{Pb}^{2+}=$ dark gray (octahedra); $\mathrm{N}=$ violet; $\mathrm{C}=$ black; $\mathrm{H}$ = white. 


\section{Identification and refinement of the $\mathrm{Cs}-\mathrm{Pb}-\mathrm{Cl}-\mathrm{I}$ nanoplatelets structure}

We then turned our attention to mixed-halide $\mathrm{Cl}-\mathrm{I}$ nanoplatelets, a system that offers an increased structural complexity due to the presence of two different anions which can, in principle, can share the same positions within the structures of cesium lead halides. This combination of halides is appealing for a diffraction analysis due to the large difference in the electron densities between $\mathrm{Cl}^{-}$and $\mathrm{I}^{-}$, which makes it easier to tell them apart, and because the different ionic radii favors the segregation of halides, inducing the formation of the $\mathrm{Cs}_{2} \mathrm{PbCl}_{2} \mathrm{I}_{2}$ Ruddlesden-Popper phase. ${ }^{50}$ Only a few reports were published on nanocrystals of this phase, and a detailed characterization of their structure is lacking to date. ${ }^{48,49,51,52}$

The $\mathrm{Cs}-\mathrm{Pb}-\mathrm{Cl}-\mathrm{I}$ nanoplatelets were prepared by a modification of the protocol used for $\mathrm{Cs}-\mathrm{Pb}-\mathrm{Br}$ nanoplatelets. The modifications consisted in using a doubled amount of Cs-oleate and in injecting a 1:1 mixture of benzoyl chloride and iodide at $50^{\circ} \mathrm{C}$. Upon injection, the solution immediately turned red, followed by a color transition to light red-orange after a minute of growth, and to pale yellow upon addition of ethyl acetate antisolvent during the nanoplatelet isolation. The synthesized particles were analyzed by absorption spectroscopy and TEM (Figure 3). The absorption spectrum features a strong excitonic peak at $3.12 \mathrm{eV}$, compatible with that reported in the past for $\mathrm{Cs}_{2} \mathrm{PbCl}_{2} \mathrm{I}_{2}$ nanoplatelets. ${ }^{48,49} \mathrm{It}$ is worth noting that in $\mathrm{Cs}_{2} \mathrm{PbCl}_{2} \mathrm{I}_{2}$ the exciton absorption position is weakly dependent on the thickness of nanocrystals, as the exciton peak in the bulk is red-shifted by only $0.08 \mathrm{eV}(3.04 \mathrm{eV})$. This is because the $\left[\mathrm{PbX}_{6}\right]^{4-}$ octahedra are disconnected along the $c$ crystallographic axis and behave as individually confined systems regardless of the thickness of the crystal. ${ }^{48}$ Therefore, the thickness of the platelets had to be inferred directly from the diffraction pattern. The sample was self-assembled into a multilayer film and analyzed through X-ray diffraction, producing a pattern that appeared very different from the case of bromine-based platelets: the most intense group of fringes was found at a different position in the $q$-scale $\left(\sim 2 \AA^{-1}\right)$, and was less broad, suggesting thicker platelets than in the previous case. Furthermore, we identified two diffraction features that did not belong to the general periodicity of the multilayer, located at $q=\sim 1.12 \AA^{-1}$ and $q=\sim 2.22 \AA^{-1}$. The $\{100\}$ and $\{200\}$ Bragg peaks of pseudocubic $\mathrm{CsPbCl}_{3}{ }^{53,54}$ provided a potential match for these diffraction features, possibly indicating an impurity of this compound in the sample. Since their overlap with the $\mathrm{Cs}-\mathrm{Pb}-\mathrm{Cl}-\mathrm{I}$ diffraction features is minimal, we decided not to subtract them to minimize unnecessary data processing.

In order to find a suitable starting model for the refinement of $\mathrm{Cs}-\mathrm{Pb}-\mathrm{Cl}-\mathrm{I}$ nanoplatelets, we tested several possible structures, obtained by slicing the reported bulk structure, until we found a good match with $3\left[\mathrm{PbX}_{6}\right]^{4-}$ octahedra thick, $\mathrm{I}-/ \mathrm{NH}_{4}-\mathrm{R}^{+}$terminated Ruddlesden Popper nanoplatelets (see $\mathrm{SI}$, Section S7). Starting from this preliminary model, we refined the atomic coordinates and occupancies of the atoms on the surface. The platelets were found to be $25.11 \pm 0.03 \AA$ thick, and were separated by an interparticle layer of $30.25 \pm 0.08 \AA$ with a stacking disorder parameter of $0.41 \pm 0.07 \AA$. This corresponds to an overall multilayer periodicity of $55.36 \pm 0.08 \AA$. Like $\mathrm{Cs}-\mathrm{Pb}-\mathrm{Br}$ platelets, the crystal structure was slightly expanded along the thickness of the platelets ( $\mathrm{Pb}-\mathrm{Pb}$ vertical distance $=9.58 \AA$ vs $9.44 \AA$ published, $\left.{ }^{50}+1.46 \%\right)$. The surface coverage was found to be $90 \pm 6 \%$, higher than that measured for pure bromide nanoplatelets.

Thanks to the excellent quality of the diffractogram, we could also refine the coordinates of the atomic planes within the platelets. Interestingly, the $\mathrm{Cs}^{+}$and $\mathrm{I}^{-}$ions were found to be almost coplanar $\left(\mid \mathrm{z}_{\mathrm{cs}^{-}}\right.$ $z_{1} \mid=0.17 \pm 0.15 \AA$ ), whereas in the structure reported for bulk $\left.\mathrm{Cs}_{2} \mathrm{PbCl}_{2}\right|_{2}$ they are shifted by $0.68 \AA$, therefore forming strongly staggered planes. This is most likely an effect of the expansion along the thin direction, that stretches the structure forcing the alignment. To conclude, we remark that the fit produced excellent results even if the iodide and chloride atoms were assigned to strictly different crystallographic positions, without 
the need of considering mixed occupancies. This indicates that the two halides segregate completely within the nanoplatelets, a result expected based on the crystal structure of bulk $\mathrm{Cs}_{2} \mathrm{PbCl}_{2} \mathrm{I}_{2}$, but not obvious for ultrathin nanoplatelets exposed to a reaction environment containing an excess of both $\mathrm{Cl}^{-}$and $\mathrm{I}^{-}$ions. The proposed structural model corresponds to a composition of $\mathrm{Cs}: \mathrm{Pb}: \mathrm{Cl}: I=4: 3: 6: 5.8$. The experimental composition of the sample was found to be $\mathrm{Cs}: \mathrm{Pb}: \mathrm{Cl}: \mathrm{I}=4.8: 4.3: 9.2: 5.8$ by SEM-EDS (see SI, Section S8). That composition leaves $\mathrm{Cs}: \mathrm{Pb}: \mathrm{Cl}=0.8: 1.3: 3.2$ ratio after subtracting the predicted ratio. Such residue is consistent with having an $\mathrm{CsPbCl}_{3}$ impurity in the sample as was spotted by XRD.

\section{Discussion}

The self-assembled periodic stacks of cesium lead halide nanoplatelets we investigated can be considered, at least along the probed assembly direction, the analogue of a hybrid organic-inorganic single crystal. In fact, in such mesostructures the nanoplatelets, their surface layers, and the organic ligands in between them become the structural motif that is repeated by the periodicity of the multilayer. In analogy with the refinement procedures adopted for conventional crystals, the structure of the multilayer can be refined through a multiparametric fit of its diffraction pattern. Given a starting structural model that qualitatively matches the experimental data, the refinement procedure allows to measure the multilayer periodicity, its structural structural disorder, and the interparticle distance. Furthermore, it gives access to the accurate determination of the nanoplatelets thickness, crystal structure, stoichiometry and even surface passivation. As a result, the method is able to provide insights into the surface composition, such as the determination of the surface coverage of nanoplatelets and the absence of oleic acid in their passivation layer that we demonstrated in this work. Furthermore, it enables a detailed comparison between the structure of nanoplatelets and that of the corresponding bulk phases, as in the case $\mathrm{Cs}_{2} \mathrm{Pbl}_{2} \mathrm{Cl}_{2}$, where the $\mathrm{Cs}^{+}$ and $\mathrm{I}$ ions were found to be coplanar in nanoplatelets while they are misaligned in bulk crystals (see Figure $3 d$, inset).

While relevant per se, we predict that such insights will become even more significant for the rapidly growing field of self-assembled halide perovskite superstructures, ${ }^{5,18,24,25,55-57}$ where the methodology we have developed might complement or even outmatch more elaborate characterization tools within the limits imposed by probing a single direction. In fact, the Multilayer Diffraction approach we adopted provides a large amount of detailed information while relying solely on a simple and widely accessible $\theta: 2 \theta$ out-of-plane diffraction experiment. Such experimental geometry is often applied for in situ investigations, and if applied to nanoplatelet multilayers would give the opportunity to monitor their structural response under under external stimuli such as illumination, charge injection, temperature or pressure gradients, and exchange or intercalation of chemical species.

Finally, by performing a wide-range literature search we identified a large number of published diffraction patterns that could be analyzed with a Multilayer Diffraction approach. Those are recognizable by the equally spaced diffraction peaks whose intensity shows a collective trend (e.g., decreasing in intensity towards higher $q$ or, as in this work, organized in broad groups with maximum intensity at their centre). Such diffraction patterns often reported for other metal-halides, both in the form of colloidal bidimensional nanostructures or of bulk layered phases. ${ }^{58-60}$ However, we also found several other examples for metal oxides, ${ }^{61-64}$ metal hydroxides, ${ }^{65,66}$ natural layered silicates, ${ }^{67}$ and MXenes, ${ }^{68,69}$ both in the form of colloidal bidimensional nanostructures or layered bulk crystals. To these materials, and any other that exhibits a 
tendency to form stacks, the Multilayer Diffraction approach represents a new opportunity to gain deeper insight into their compositional, structural, and surface-related properties.

\section{Methods}

\section{Chemicals.}

Lead(II) acetate trihydrate (99.999\%, Sigma-Aldrich), cesium acetate (99.99\%, Sigma-Aldrich), benzoyl chloride (Bz-Cl, 99\%, Sigma-Aldrich), benzoyl bromide (97\%, Sigma-Aldrich), sodium iodide (99.5\%, Sigma-Aldrich), decane (anhydrous, $\geq 99 \%$, Sigma-Aldrich), oleylamine (technical grade, $70 \%$, Sigma-Aldrich), oleic acid (technical grade, 90\%, Sigma-Aldrich), octylamine (99\%, Sigma-Aldrich), erucic acid (analytical standard, $\geq 99.0 \%$, Sigma-Aldrich Supelco ${ }^{\circledR}$ ), hexane (puriss. p.a., ACS reagent, Sigma-Aldrich), ethyl acetate (puriss. p.a., ACS reagent, Sigma-Aldrich). All chemicals were used without further purification.

\section{Synthesis of $\mathrm{Cs}-\mathrm{Pb}-\mathrm{X}$ nanoplatelets.}

All the nanoplatelets were prepared according to the same general procedure, which is here exemplified for the case of oleylammonium-capped $\mathrm{Cs}-\mathrm{Pb}-\mathrm{Br}$ nanoplatelets. The specific conditions applied for each sample discussed in this work are detailed in the SI, Table S1. First, a solution of ligands ( $120 \mu \mathrm{L}$ of oleylamine and $160 \mu \mathrm{L}$ of oleic acid) in $2 \mathrm{ml}$ of decane was heated up to $100^{\circ} \mathrm{C}$ inside an $8 \mathrm{ml}$ glass vial. Second, the metal carboxylates ( $60 \mu \mathrm{L}$ of Pb-oleate and $20 \mu \mathrm{L}$ of $\mathrm{Cs}$-Oleate) were pre-heated to the same temperature to achieve a good homogeneity and a decent fluidity, and added to the solution by dipping and flushing the micropipette tips inside the liquid several times to ensure a quantitative release. Finally, the benzoyl halide (20 $\mu \mathrm{L}$ of $\mathrm{Bz}-\mathrm{Br}$ ) was injected in the solution at $100^{\circ} \mathrm{C}$, triggering the immediate nucleation of the nanoplatelets. After a growth time of $1 \mathrm{~min}$, the reaction was quenched by immersing the vial in water at room temperature. The resulting colloidal suspension was destabilized by adding ethyl acetate until it turned cloudy ( $4 \mathrm{ml})$. The particles were recovered by centrifuging at $4000 \mathrm{rpm}$ for 2 minutes and were resuspended in $1.2 \mathrm{ml}$ of hexane. The so-obtained suspension was filtered with a $20 \mu \mathrm{m}$ PTFE syringe filter and stored in a closed vial.

\section{Benzoyl iodide preparation.}

Benzoyl iodide (Bz-l) was prepared by mixing $1.4 \mathrm{ml}$ of benzoyl chloride and $3.0 \mathrm{~g}$ of sodium iodide at $\sim 75^{\circ} \mathrm{C}$ overnight inside a $\mathrm{N}_{2}$-filled glovebox. The liquid was recovered with a syringe, filtered with a PTFE 0.2 $\mu \mathrm{m}$ filter, and collected inside a vial. After a few hours at room temperature some solid, likely a sodium halide, formed on the vial walls and was discarded by repeating the filtration process. The reaction product was an orange-red liquid, that could be stored inside a glovebox in a dark vial for more than one month without any appreciable change in its aspect or reactivity.

\section{Preparation of metal carboxylates.}

Lead oleate was prepared by mixing $379 \mathrm{mg}(1 \mathrm{mmol})$ of lead acetate trihydrate with $1.5 \mathrm{ml}$ of oleic acid at $100^{\circ} \mathrm{C}$ for $3 \mathrm{~h}$. The process was carried out under mild vacuum to ease the volatilization of acetic acid. Cesium oleate was prepared by mixing $192 \mathrm{mg}(1 \mathrm{mmol})$ of cesium acetate with $1.0 \mathrm{ml}$ of oleic acid at $100^{\circ} \mathrm{C}$ for $\sim 1 \mathrm{~h}$. The process was carried out under mild vacuum to ease the volatilization of acetic acid. Both processes yielded dense yellow liquids which readily solidified when cooled at room temperature. Metal 
oleates were pre-heated to $100^{\circ} \mathrm{C}$ and stirred for a few minutes before taking any aliquot, to ensure the compositional homogeneity of the precursor. Lead and cesium erucates were prepared alike, simply replacing oleic acid with an equimolar amount of erucic acid. For Cs-erucate $1.07 \mathrm{~g}$ were used, while for $\mathrm{Pb}$ erucate 1.61 were used.

\section{Preparation of nanoplatelet films.}

The films for the Multilayer Diffraction experiments were prepared by diluting with hexane the nanoparticles solutions obtained as described above by a factor of 2-5. After the dilution, $50 \mu \mathrm{L}$ of solution were carefully deposited on a $1 \times 1 \mathrm{~cm}$ silicon wafer (Ted Pella, Inc., <100> orientation) placed inside a glass Petri dish (inner volume $\sim 25 \mathrm{~cm}^{3}$ ). Then, the Petri dish was closed with its glass lid, and a chink in between the edge and the lid was created by adding a small piece of folded aluminum foil. The solution was let drying completely until a homogeneous and iridescent film was formed: the process required about 2-5 minutes. If the film appeared opaque and not iridescent, the solution was further diluted, and the process repeated. If the film appeared too thin or invisible, a more concentrated solution was used instead. Figure S3 shows one successfully prepared film as seen from above. A 1-mm thick glass slide placed underneath the silicon to tilt it at an angle of about $10-15^{\circ}$ helps in getting more homogeneous films on at least one region of the substrate.

\section{Diffraction data collection and processing.}

The diffraction patterns of the nanoplatelet films were collected with a Panalytical Empyrean diffractometer in a parallel-beam configuration, equipped with a $1.8 \mathrm{~kW} \mathrm{Cu}$ K $\alpha$ ceramic X-ray tube operating at $45 \mathrm{kV}, 1 \mathrm{~mm}$ wide incident and receiving slits, and a $40 \mathrm{~mA}$ PIXcel3D $2 \times 2$ bidimensional detector. While the bidimensional detector is not a strict requirement for the application of the Multilayer Diffraction method, it can help better visualizing the data and recognizing the signals coming from multilayer stacks of nanoplatelets from those produced by other sources (e.g. the substrate or misaligned particles). In fact, multilayer diffraction signals appear in the form of vertical straight stripes. Instead, the substrate produces clusters of sharp and intense spots, while misaligned nanoparticles produce arc-shaped signals. The bidimensional data were integrated over a rectangular sector, chosen to exclude or minimize the contribution of other signals than those coming from the multilayers. Figure S4a shows a representative bidimensional diffractogram containing signals from the multilayer film and the substrate, and some residual misaligned particles, the rectangular sector selected for the integration, shown in yellow.

The instrumental background was measured on a clean silicon wafer and integrated under the same conditions applied for the sample. Then, its 1D profile was modeled into a spline using the MagicPlot software version 2.9.2, and subtracted from the sample diffractogram after being rescaled if needed. A background was considered well-paired with the experimental data if it could adequately describe the diffracted intensity found in regions far from intense multilayer diffraction fringes. Figure S4b shows an example of 1D diffractogram as obtained after the integration of the 2D raw data, together with a properly scaled background ready for the subtraction.

In the event that not all the spurious signals could be excluded during the integration step, a subtraction was attempted. If the extra signal was far from any multilayer fringe, it was simply described as a sum of gaussians, subtracted, and the pattern was locally smoothed by a moving average algorithm. If it was closed or overlapped with the multilayer signal, instead, a different approach was taken. First, the nearby multilayer fringes were fitted with a gaussian profile to recover their position and broadening. Then, the 
position and broadening of the multilayer fringe suffering from the overlap was inferred from those of the neighboring fringes. Finally, the pattern region affected by the overlap was fitted with the sum of a gaussian peak, representing the multilayer fringe and having its position and broadening fixed, and a sum of additional peaks describing the additional, unwanted signals. Those were subtracted, and the pattern was then ready for the fit. This data treatment was performed only if the spurious signal was reasonably weak if compared with the neighboring multilayer fringes. If not, the pattern was fitted as it was (as for Figure 3 in the main text), or simply discarded. Figure S4c shows a diffraction pattern after the subtraction of the background and of some spurious signals, shown in red.

The application of the Lorentz-Polarization-Absorption (LPA) correction is the last treatment performed on the diffractogram before proceeding with the Multilayer Diffraction fit. This step is needed to compensate for the geometrical and instrumental contributions to the diffracted intensity, in order to transform the experimental diffractogram into an experimental measurement of the square modulus of the multilayer structure factor $\left|F_{M L}(q)\right|^{2}$. This is the mathematical entity computed by our fitting algorithm and directly relatable to the structure of the multilayer. The LPA correction is a function of the beam incidence angle $\theta$, it must be applied to each experimental point of the diffractogram, and is described by Equation $3:{ }^{26}$

$$
I_{L P A}=I_{0} \cdot\left[\frac{\left(1-e^{-\frac{2 \mu}{\sin (\theta)}}\right) \cdot\left(1+\cos ^{2}\left(2 \theta_{m}\right) \cdot \cos ^{2}(2 \theta)\right)}{\sin (2 \theta)}\right] \quad \text { Eq. } 3
$$

Where $I_{0}$ is the intensity measured experimentally (after the subtraction of background), $\mu$ is the absorption coefficient of the material, $\tau$ is the film thickness, and $\vartheta_{m}$ is the Goebel mirror Bragg angle of the diffractometer $\left(1^{\circ}\right.$ in our case). The product $\mu \tau$ is responsible for correction the effects of the $\mathrm{X}$-rays absorption occurring in thick films. However, all our samples belonged to the thin film regime, where the absorption is negligible and $\mu \tau \rightarrow 0$. Therefore, it was set constant to a small enough value in the fitting algorithm ( $\mu \tau=0.00001)$. Smaller values for the product $\mu \tau$ would lead our algorithm to crash. The pattern resulting from the LPA-correction is shown in Figure S4d.

\section{Outline of the Multilayer Diffraction method.}

The fitting algorithm presented in this work is an evolution of the one we presented in our previous work on the diffraction analysis of nanocrystal superlattices, to which we refer the reader for additional details. ${ }^{24}$ In short, the algorithm computes the square modulus of the multilayer structure factor $\left|F_{M L}(q)\right|^{2}$ based on a structural model of the multilayer, which includes both instrumental and structural parameters, Our algorithm refines these parameters by comparing the simulated $\left|F_{M L}(q)\right|^{2}$ with the experimental data processed as illustrated above, via the nonlinear least square minimization of a cost function. The number and nature of the optimizable parameters was editable, to allow for the maximum flexibility of the code. Those can be divided into three groups, whose impact on the simulation is described below.

The instrumental parameters describe the contribution of the diffractometer to the measured diffraction profile. These account for the incident X-ray wavelength, the instrumental broadening of the diffraction signal, the Goebel mirror incidence angle and for possible misalignments of the sample during the measurement. The multilayer parameters describe the structure of the multilayer at super-atomic scale. These mostly impact the periodicity and broadening of the diffraction fringes in the diffractogram, and account for the nanoplatelet thickness, the interplatelet distance, the structural disorder of the multilayer, and the electron density of the organic layers in between the nanoplatelets. The nanoplatelet parameters 
describe the atomistic structure of the nanoplatelets. These contribute to the simulation of the nanoplatelet structure factor, and mostly impact the integrated areas of the diffraction fringes in the diffractograms. Their number can be changed to meet the requirements of the specific experiments, and constraints can be imposed during the fit to account for relationships in between them. In general, these parameters describe the vertical coordinate of each atomic layer inside the nanoplatelet, the elements each layer contains and the fractional occupancy of each atom in the layer. A more detailed breakdown of the meaning and role of each parameter is provided in the SI, Section S3.

Based on these parameters, the algorithm first computes the structure factors of both the nanoplatelets ( $F_{N P}$, by applying the Equation 2 ), and the interplatelet organic layer $F_{O L}$. The latter is modeled for simplicity as an amorphous layer of carbon atoms, whose density is estimated a priori based on the density of long-chain liquid hydrocarbons. For further details see $\mathrm{Sl}$, section $\mathrm{S} 5$. The structure factor of the organic layer is computed according to Equation 4:

$$
F_{O L}(q)=\rho_{C} \cdot f_{C}(q) \cdot \int_{0}^{L} e^{-i q \cdot z} d z=\frac{\rho_{C} \cdot f_{C}(q)}{i q} \cdot\left(e^{i q \cdot L}-1\right) \quad \text { Eq. } 4
$$

Where $\rho_{c}$ is the linear density of carbon atoms expressed in atoms / $\AA$ formula unit, $f_{C}$ is the atomic form factor of Carbon, and $L$ is the interparticle distance. Once $F_{N P}$ and $F_{O L}$ have been calculated, the algorithm proceeds with computing the multilayer structure factor $F_{M L}$ first and of the multilayer diffraction pattern $\left|F_{M L}(q)\right|^{2}$ then, according to the equations derived by Fullerton et al. (here not shown for brevity). ${ }^{26}$ As a last step, the algorithm simulates the effects of the instrumental broadening on the just obtained diffraction pattern, and then compares the obtained profile with the experimental one. This comparison is repeated over and over while the program optimizes all the fittable parameters by minimizing the mismatch in between the experimental and the simulated pattern.

To conclude, the values and the standard deviations reported in this article for each parameter are the result of a bootstrap analysis performed on the fit. In short, the algorithm adds to the experimental dataset a gaussian noise estimated over the variance of the experimentally measured diffracted intensity, and then performs the fit. This procedure is repeated 300 times, after that the value and standard deviation of each fittable parameter is expressed as the average and standard deviation of the values found, at each iteration, for said parameter. For further details on the bootstrap procedure and the estimation of the experimental data variance please refer to our previous publication. ${ }^{24}$

\section{Other characterization techniques.}

The absorption spectra from colloidal suspensions of nanoplatelets were acquired on Cary300 spectrophotometer. The photoluminescence spectra were collected instead on a Cary Eclipse spectrofluorometer. Low magnification transmission electron microscopy (TEM) and selected-area electron diffraction (SAED) images were acquired on a JEOL JEM-1011 microscope equipped with a thermionic gun at an accelerating voltage of $100 \mathrm{kV}$. The samples were prepared by depositing a diluted suspension of nanoparticles on a 200-mesh carbon-coated copper grids. Energy dispersive X-ray spectroscopy (EDS) measurements were performed at $25 \mathrm{kV}$ on a JEOL JSM-6490LA scanning electron microscope (SEM). All the crystal structure models presented in this work have been built using VESTA , ver. 3.4.6. ${ }^{70}$

\section{Data availability.}


All the diffraction patterns analysed in this work are available (after background subtraction, substrate/spurious signal subtraction, and $2 \theta \rightarrow q$ conversion) as a part of the Supplementary Material in the form of .csv q/intensity spreadsheets. The Multilayer Diffraction algorithm and the fitting results are available as a part of the Supplementary Material in the form of .ipynb Jupyter Lab notebooks (one for each diffraction pattern) accompanied by the MD_Core.py file (required for running the notebooks). All the other data are available from the corresponding authors upon reasonable request.

\section{Akcnowledgements.}

We thank the Materials Characterization Facility and Electron Microscopy Facility at the Fondazione Istituto Italiano di Tecnologia for use of XRD and TEM/SEM equipment and technical support, Mr. Sergio Marras for support with diffraction experiments and discussion, Dr. Mirko Prato for helpful discussions, and Dr. Jakob Dahl and Mr. Xingzhi Wang for the discussion of the nanoplatelet synthesis.

\section{Author contributions.}

S.T. - conceptualization, data curation, formal analysis, investigation (synthesis and diffraction experiments), methodology, software (script development and validation), writing (original draft, review and editing), visualization (figures); D.B. - conceptualization, data curation, formal analysis, investigation (SEMEDS), methodology, validation, writing (review and editing), supervision; C.G. - conceptualization, methodology, supervision; L.M. - conceptualization, supervision, writing (review and editing). Author contributions are assigned using CRediT taxonomy (https://casrai.org/credit/).

\section{Competing interests.}

The authors declare no competing interests.

\section{Additional information}

Supplementary information is available for this paper at \&\&\&. Experimental XRD diffractograms and corresponding Jupyter Notebooks; Python script containing the Multilayer Diffraction fitting algorithm (ZIP). Experimental conditions for the synthesis of $\mathrm{Cs}-\mathrm{Pb}-\mathrm{X}$ nanoplatelets and detailed synthetic protocol for $\mathrm{Cs}-\mathrm{Pb}$ $\mathrm{Br}$ nanoplatelet synthesis; description of the background and interferent signals subtraction; outline of the Multilayer Diffraction algorithm; preliminary simulations of the $\mathrm{Cs}-\mathrm{Pb}-\mathrm{X}$ diffraction patterns; parametrization of the $\mathrm{Cs}-\mathrm{Pb}-\mathrm{X}$ nanoplatelet structures and fit results; additional comments on the surface passivation of Cs$\mathrm{Pb}-\mathrm{X}$ nanoplatelets; SEM-EDS compositional analyses of Cs-Pb-X nanoplatelets. 


\section{References}

(1) Sichert, J. A.; Tong, Y.; Mutz, N.; Vollmer, M.; Fischer, S.; Milowska, K. Z.; García Cortadella, R.; Nickel, B.; Cardenas-Daw, C.; Stolarczyk, J. K.; Urban, A. S.; Feldmann, J. Quantum Size Effect in Organometal Halide Perovskite Nanoplatelets. Nano Lett. 2015, 15, 6521-6527.

(2) Weidman, M. C.; Goodman, A. J.; Tisdale, W. A. Colloidal Halide Perovskite Nanoplatelets: An Exciting New Class of Semiconductor Nanomaterials. Chem. Mater. 2017, 29, 5019-5030.

(3) Akkerman, Q. A.; Motti, S. G.; Srimath Kandada, A. R.; Mosconi, E.; D'Innocenzo, V.; Bertoni, G.; Marras, S.; Kamino, B. A.; Miranda, L.; De Angelis, F.; Petrozza, A.; Prato, M.; Manna, L. Solution Synthesis Approach to Colloidal Cesium Lead Halide Perovskite Nanoplatelets with Monolayer-Level Thickness Control. J. Am. Chem. Soc. 2016, 138, 1010-1016.

(4) Gao, F.; Yang, W.; Liu, X.; Li, Y.; Liu, W.; Xu, H.; Liu, Y. Highly Stable and Luminescent Silica-Coated Perovskite Quantum Dots at Nanoscale-Particle Level via Nonpolar Solvent Synthesis. Chem. Eng. J. 2021, 407, 128001.

(5) Jagielski, J.; Solari, S. F.; Jordan, L.; Scullion, D.; Blülle, B.; Li, Y. T.; Krumeich, F.; Chiu, Y. C.; Ruhstaller, B.; Santos, E. J. G.; Shih, C. J. Scalable Photonic Sources Using Two-Dimensional Lead Halide Perovskite Superlattices. Nat. Commun. 2020, 11, 1-9.

(6) Morgenstern, T.; Lampe, C.; Naujoks, T.; Jurow, M.; Liu, Y.; Urban, A. S.; Brütting, W. Elucidating the Performance Limits of Perovskite Nanocrystal Light Emitting Diodes. J. Lumin. 2020, 220, 116939.

(7) Cui, J.; Liu, Y.; Deng, Y.; Lin, C.; Fang, Z.; Xiang, C.; Bai, P.; Du, K.; Zuo, X.; Wen, K.; Gong, S.; He, H.; Ye, Z.; Gao, Y.; Tian, H.; Zhao, B.; Wang, J.; Jin, Y. Efficient Light-Emitting Diodes Based on Oriented Perovskite Nanoplatelets. arXiv 2020, arXiv:2006.07611.

(8) Bertolotti, F.; Nedelcu, G.; Vivani, A.; Cervellino, A.; Masciocchi, N.; Guagliardi, A.; Kovalenko, M. V. Crystal Structure, Morphology, and Surface Termination of Cyan-Emissive, Six-Monolayers-Thick $\mathrm{CsPbBr}_{3}$ Nanoplatelets from X-Ray Total Scattering. ACS Nano 2019, 13, 14294-14307.

(9) Akkerman, Q. A.; Martín-García, B.; Buha, J.; Almeida, G.; Toso, S.; Marras, S.; Bonaccorso, F.; Petralanda, U.; Infante, I.; Manna, L. Ultrathin Orthorhombic PbS Nanosheets. Chem. Mater. 2019, $31,8145-8153$.

(10) Chen, D.; Gao, Y.; Chen, Y.; Ren, Y.; Peng, X. Structure Identification of Two-Dimensional Colloidal Semiconductor Nanocrystals with Atomic Flat Basal Planes. Nano Lett. 2015, 15, 4477-4482.

(11) Zhang, Y.; Zhang, H.; Chen, D.; Sun, C.-J.; Ren, Y.; Jiang, J.; Wang, L.; Li, Z.; Peng, X. Engineering of Exciton Spatial Distribution in CdS Nanoplatelets. Nano Lett. 2021, 19, 48.

(12) Bekenstein, Y.; Koscher, B. A.; Eaton, S. W.; Yang, P.; Alivisatos, A. P. Highly Luminescent Colloidal Nanoplates of Perovskite Cesium Lead Halide and Their Oriented Assemblies. J. Am. Chem. Soc. 2015, 137, 16008-16011.

(13) Rakita, Y.; Cohen, S. R.; Kedem, N. K.; Hodes, G.; Cahen, D. Mechanical Properties of $\mathrm{APbX}_{3}(\mathrm{~A}=\mathrm{Cs}$ or $\mathrm{CH}_{3} \mathrm{NH}_{3} ; \mathrm{X}=\mathrm{i}$ or $\mathrm{Br}$ ) Perovskite Single Crystals. MRS Commun. 2015, 5, 623-629.

(14) Ferreira, A. C.; Létoublon, A.; Paofai, S.; Raymond, S.; Ecolivet, C.; Rufflé, B.; Cordier, S.; Katan, C.; Saidaminov, M. I.; Zhumekenov, A. A.; Bakr, O. M.; Even, J.; Bourges, P. Elastic Softness of Hybrid Lead Halide Perovskites. Phys. Rev. Lett. 2018, 121, 085502.

(15) Chu, W.; Saidi, W. A.; Zhao, J.; Prezhdo, O. V. Soft Lattice and Defect Covalency Rationalize Tolerance of $\beta$-CsPbl ${ }_{3}$ Perovskite Solar Cells to Native Defects. Angew. Chemie - Int. Ed. 2020, 59, 6435-6441.

Lai, M.; Obliger, A.; Lu, D.; Kley, C. S.; Bischak, C. G.; Kong, Q.; Lei, T.; Dou, L.; Ginsberg, N. S.; 
Limmer, D. T.; Yang, P. Intrinsic Anion Diffusivity in Lead Halide Perovskites Is Facilitated by a Soft Lattice. Proc. Natl. Acad. Sci. U. S. A. 2018, 115, 11929-11934.

(17) Jurow, M. J.; Morgenstern, T.; Eisler, C.; Kang, J.; Penzo, E.; Do, M.; Engelmayer, M.; Osowiecki, W. T.; Bekenstein, Y.; Tassone, C.; Wang, L. W.; Alivisatos, A. P.; Brütting, W.; Liu, Y. Manipulating the Transition Dipole Moment of $\mathrm{CsPbBr}_{3}$ Perovskite Nanocrystals for Superior Optical Properties. Nano Lett. 2019, 19, 2489-2496.

(18) Kumar, S.; Marcato, T.; Krumeich, F.; Li, Y.-T.; Chiu, Y.-C.; Shih, C.-J. Two-Dimensional Nanoplatelet Superlattices Overcoming Light Outcoupling Efficiency Limit in Perovskite Quantum Dot LightEmitting Diodes. Res. Sq. 10.21203/rs.3.rs-344894/v1.

(19) Chen, J.; Zhou, Y.; Fu, Y.; Pan, J.; Mohammed, O. F.; Bakr, O. M. Oriented Halide Perovskite Nanostructures and Thin Films for Optoelectronics. Chem. Rev. 2021, 10.1021/acs.chemrev.1c00181.

(20) Gemmi, M.; Mugnaioli, E.; Gorelik, T. E.; Kolb, U.; Palatinus, L.; Boullay, P.; Hovmöller, S.; Abrahams, J. P. 3D Electron Diffraction: The Nanocrystallography Revolution. ACS Cent. Sci. 2019, 5, 1315-1329.

(21) Yu, Y.; Zhang, D.; Kisielowski, C.; Dou, L.; Kornienko, N.; Bekenstein, Y.; Wong, A. B.; Alivisatos, A. P.; Yang, P. Atomic Resolution Imaging of Halide Perovskites. Nano Lett. 2016, 16, 7530-7535.

(22) Brescia, R.; Toso, S.; Ramasse, Q.; Manna, L.; Shamsi, J.; Downing, C.; Calzolari, A.; Bertoni, G. Bandgap Determination from Individual Orthorhombic Thin Cesium Lead Bromide Nanosheets by Electron Energy-Loss Spectroscopy. Nanoscale Horizons 2020, 5, 1610-1617.

(23) Ferri, F.; Bertolotti, F.; Guagliardi, A.; Masciocchi, N. Nanoparticle Size Distribution from Inversion of Wide Angle X-Ray Total Scattering Data. Sci. Rep. 2020, 10, 12759.

(24) Toso, S.; Baranov, D.; Altamura, D.; Scattarella, F.; Dahl, J.; Wang, X.; Marras, S.; Alivisatos, A. P.; Singer, A.; Giannini, C.; Manna, L. Multilayer Diffraction Reveals That Colloidal Superlattices Approach the Structural Perfection of Single Crystals. ACS Nano 2021, 15, 6243-6256.

(25) Toso, S.; Baranov, D.; Giannini, C.; Marras, S.; Manna, L. Wide-Angle X-Ray Diffraction Evidence of Structural Coherence in $\mathrm{CsPbBr}_{3}$ Nanocrystal Superlattices. ACS Mater. Lett. 2019, 1, 272-276.

(26) Fullerton, E. E.; Schuller, I. K.; Vanderstraeten, H.; Bruynseraede, Y. Structural Refinement of Superlattices from X-Ray Diffraction. Phys. Rev. B 1992, 45, 9292-9310.

(27) Zhao, Q.; Hazarika, A.; Schelhas, L. T.; Liu, J.; Gaulding, E. A.; Li, G.; Zhang, M.; Toney, M. F.; Sercel, P. C.; Luther, J. M. Size-Dependent Lattice Structure and Confinement Properties in $\mathrm{CsPbl}_{3}$ Perovskite Nanocrystals: Negative Surface Energy for Stabilization. ACS Energy Lett. 2020, 5, 238-247.

(28) Dahl, J. C.; Wang, X.; Huang, X.; Chan, E. M.; Alivisatos, A. P. Elucidating the Weakly Reversible Cs-PbBr Perovskite Nanocrystal Reaction Network with High-Throughput Maps and Transformations. J. Am. Chem. Soc. 2020, 142, 11915-11926.

(29) Kitagawa, K. Thin-Film Thickness Profile Measurement by Three-Wavelength Interference Color Analysis. Appl. Opt. 2013, 52, 1998-2007.

(30) Warren, B. E. X-Ray Diffraction; Courier Corporation, 1990.

(31) Do, M.; Kim, I.; Kolaczkowski, M. A.; Kang, J.; Kamat, G. A.; Yuan, Z.; Barchi, N. S.; Wang, L. W.; Liu, Y.; Jurow, M. J.; Sutter-Fella, C. M. Low-Dimensional Perovskite Nanoplatelet Synthesis Using In Situ Photophysical Monitoring to Establish Controlled Growth. Nanoscale 2019, 11, 17262-17269.

(32) Bodnarchuk, M. I.; Boehme, S. C.; Ten Brinck, S.; Bernasconi, C.; Shynkarenko, Y.; Krieg, F.; Widmer, R.; Aeschlimann, B.; Günther, D.; Kovalenko, M. V.; Infante, I. Rationalizing and Controlling the Surface Structure and Electronic Passivation of Cesium Lead Halide Nanocrystals. ACS Energy Lett. 
$2019,4,63-74$.

(33) De Roo, J.; Ibáñez, M.; Geiregat, P.; Nedelcu, G.; Walravens, W.; Maes, J.; Martins, J. C.; Van Driessche, I.; Kovalenko, M. V.; Hens, Z. Highly Dynamic Ligand Binding and Light Absorption Coefficient of Cesium Lead Bromide Perovskite Nanocrystals. ACS Nano 2016, 10, 2071-2081.

(34) Ijaz, P.; Imran, M.; Soares, M. M.; Tolentino, H. C. N.; Martín-García, B.; Giannini, C.; Moreels, I.; Manna, L.; Krahne, R. Composition-, Size-, and Surface Functionalization-Dependent Optical Properties of Lead Bromide Perovskite Nanocrystals. J. Phys. Chem. Lett. 2020, 11, 2079-2085.

(35) Quarta, D.; Imran, M.; Capodilupo, A. L.; Petralanda, U.; Van Beek, B.; De Angelis, F.; Manna, L.; Infante, I.; De Trizio, L.; Giansante, C. Stable Ligand Coordination at the Surface of Colloidal CsPbBr 3 Nanocrystals. J. Phys. Chem. Lett. 2019, 10, 3715-3726.

(36) Chen, Y.; Smock, S. R.; Flintgruber, A. H.; Perras, F. A.; Brutchey, R. L.; Rossini, A. J. Surface Termination of $\mathrm{CsPbBr}_{3}$ Perovskite Quantum Dots Determined by Solid-State NMR Spectroscopy. J. Am. Chem. Soc. 2020, 142, 6117-6127.

(37) Rodová, M.; Brožek, J.; Knížek, K.; Nitsch, K. Phase Transitions in Ternary Caesium Lead Bromide. J. Therm. Anal. Calorim. 2003, 71, 667-673.

(38) Dos Reis, R.; Yang, H.; Ophus, C.; Ercius, P.; Bizarri, G.; Perrodin, D.; Shalapska, T.; Bourret, E.; Ciston, J.; Dahmen, U. Determination of the Structural Phase and Octahedral Rotation Angle in Halide Perovskites. Appl. Phys. Lett. 2018, 112, 071901.

(39) Toso, S.; Baranov, D.; Manna, L. Hidden in Plain Sight: The Overlooked Influence of the $\mathrm{Cs}^{+}$ Substructure on Transformations in Cesium Lead Halide Nanocrystals. ACS Energy Lett. 2020, 5, 3409-3414.

(40) Bertolotti, F.; Protesescu, L.; Kovalenko, M. V.; Yakunin, S.; Cervellino, A.; Billinge, S. J. L.; Terban, M. W.; Pedersen, J. S.; Masciocchi, N.; Guagliardi, A. Coherent Nanotwins and Dynamic Disorder in Cesium Lead Halide Perovskite Nanocrystals. ACS Nano 2017, 11, 3819-3831.

(41) Brennan, M. C.; Kuno, M.; Rouvimov, S. Crystal Structure of Individual $\mathrm{CsPbBr}_{3}$ Perovskite Nanocubes. Inorg. Chem. 2019, 58, 1555-1560.

(42) Dahlman, C. J.; Venkatesan, N. R.; Corona, P. T.; Kennard, R. M.; Mao, L.; Smith, N. C.; Zhang, J.; Seshadri, R.; Helgeson, M. E.; Chabinyc, M. L. Structural Evolution of Layered Hybrid Lead lodide Perovskites in Colloidal Dispersions. ACS Nano 2020, 14, 11294-11308.

(43) Beimborn, J. C.; Walther, L. R.; Wilson, K. D.; Weber, J. M. Size-Dependent Pressure-Response of the Photoluminescence of $\mathrm{CsPbBr}_{3}$ Nanocrystals. J. Phys. Chem. Lett. 2020, 11, 1975-1980.

(44) Patra, A.; Marjit, K.; Ghosh, G.; Ghosh, S.; Sain, S.; Ghosh, A. Structural Analysis and Carrier Relaxation Dynamics of 2D CsPbBr 3 Nanoplatelets. J. Phys. Chem. C 2021, 125, 12214-12223.

(45) Giannini, C.; Holy, V.; De Caro, L.; Mino, L.; Lamberti, C. Watching Nanomaterials with X-Ray Eyes: Probing Different Length Scales by Combining Scattering with Spectroscopy. Prog. Mater. Sci. 2020, 112, 100667.

(46) Smith, M. D.; Connor, B. A.; Karunadasa, H. I. Tuning the Luminescence of Layered Halide Perovskites. Chem. Rev. 2019, 119, 3104-3139.

(47) Li, X.; Hoffman, J. M.; Kanatzidis, M. G. The 2D Halide Perovskite Rulebook: How the Spacer Influences Everything from the Structure to Optoelectronic Device Efficiency. Chem. Rev. 2021, 121, 2230-2291.

(48) Akkerman, Q. A.; Bladt, E.; Petralanda, U.; Dang, Z.; Sartori, E.; Baranov, D.; Abdelhady, A. L.; Infante, I.; Bals, S.; Manna, L. Fully Inorganic Ruddlesden-Popper Double Cl-I and Triple Cl-Br-I Lead Halide 
Perovskite Nanocrystals. Chem. Mater. 2019, 31, 2182-2190.

(49) Dutta, A.; Behera, R. K.; Deb, S.; Baitalik, S.; Pradhan, N. Doping Mn(II) in All-Inorganic RuddlesdenPopper Phase of Tetragonal $\mathrm{Cs}_{2} \mathrm{PbCl}_{2} \mathrm{I}_{2}$ Perovskite Nanoplatelets. J. Phys. Chem. Lett. 2019, 10, $1954-$ 1959.

(50) Li, J.; Yu, Q.; He, Y.; Stoumpos, C. C.; Niu, G.; Trimarchi, G. G.; Guo, H.; Dong, G.; Wang, D.; Wang, L.; Kanatzidis, M. G. $\mathrm{Cs}_{2} \mathrm{PbI}_{2} \mathrm{Cl}_{2}$, All-Inorganic Two-Dimensional Ruddlesden-Popper Mixed Halide Perovskite with Optoelectronic Response. J. Am. Chem. Soc. 2018, 140, 11085-11090.

(51) Yang, S.; Liu, W.; Han, Y.; Liu, Z.; Zhao, W.; Duan, C.; Che, Y.; Gu, H.; Li, Y.; Liu, S. (Frank). 2D $\mathrm{Cs}_{2} \mathrm{Pbl}_{2} \mathrm{Cl}_{2}$ Nanosheets for Holistic Passivation of Inorganic $\mathrm{CsPbl}_{2} \mathrm{Br}$ Perovskite Solar Cells for Improved Efficiency and Stability. Adv. Energy Mater. 2020, 10, 2002882.

(52) Acharyya, P.; Maji, K.; Kundu, K.; Biswas, K. 2D Nanoplates and Scaled-Up Bulk Polycrystals of Ruddlesden-Popper $\mathrm{Cs}_{2} \mathrm{Pbl}_{2} \mathrm{Cl}_{2}$ for Optoelectronic Applications. ACS Appl. Nano Mater. 2020, 3, 877886.

(53) Ma, J. P.; Yin, J.; Chen, Y. M.; Zhao, Q.; Zhou, Y.; Li, H.; Kuroiwa, Y.; Moriyoshi, C.; Li, Z. Y.; Bakr, O. M.; Mohammed, O. F.; Sun, H. T. Defect-Triggered Phase Transition in Cesium Lead Halide Perovskite Nanocrystals. ACS Mater. Lett. 2019, 1, 185-191.

(54) Linaburg, M. R.; McClure, E. T.; Majher, J. D.; Woodward, P. M. $\mathrm{Cs}_{1-x} \mathrm{Rb}_{\mathrm{x}} \mathrm{PbCl}_{3}$ and $\mathrm{Cs}_{1-\mathrm{x}} \mathrm{Rb}_{\mathrm{x}} \mathrm{PbBr}_{3} \mathrm{Solid}$ Solutions: Understanding Octahedral Tilting in Lead Halide Perovskites. Chem. Mater. 2017, 29, 3507-3514.

(55) Rainò, G.; Becker, M. A.; Bodnarchuk, M. I.; Mahrt, R. F.; Kovalenko, M. V.; Stöferle, T. Superfluorescence from Lead Halide Perovskite Quantum Dot Superlattices. Nature 2018, 563, 671675.

(56) Cherniukh, I.; Rainò, G.; Stöferle, T.; Burian, M.; Travesset, A.; Naumenko, D.; Amenitsch, H.; Erni, R.; Mahrt, R. F.; Bodnarchuk, M. I.; Kovalenko, M. V. Perovskite-Type Superlattices From Lead Halide Perovskite Nanocubes. Nature 2021, 593, 535-542.

(57) Jagielski, J.; Kumar, S.; Wang, M.; Scullion, D.; Lawrence, R.; Li, Y. T.; Yakunin, S.; Tian, T.; Kovalenko, M. V.; Chiu, Y. C.; Santos, E. J. G.; Lin, S.; Shih, C. J. Aggregation-Induced Emission in Lamellar Solids of Colloidal Perovskite Quantum Wells. Sci. Adv. 2017, 3, eaaq0208.

(58) Klein, E.; Black, A.; Tokmak, Ö.; Strelow, C.; Lesyuk, R.; Klinke, C. Micron-Size Two-Dimensional Methylammonium Lead Halide Perovskites. ACS Nano 2019, 13, 6955-6962.

(59) Peng, S.; Wen, Z.; Ye, T.; Xiao, X.; Wang, K.; Xia, J.; Sun, J.; Zhang, T.; Mei, G.; Liu, H.; Xu, B.; Li, X.; Chen, R.; Xing, G.; Wang, K.; Tang, Z. Effective Surface Ligand-Concentration Tuning of Deep-Blue Luminescent $\mathrm{FAPbBr}_{3}$ Nanoplatelets with Enhanced Stability and Charge Transport. ACS Appl. Mater. Interfaces 2020, 12, 31863-31874.

(60) Walters, G.; Wei, M.; Voznyy, O.; Quintero-Bermudez, R.; Kiani, A.; Smilgies, D.-M.; Munir, R.; Amassian, A.; Hoogland, S.; Sargent, E. The Quantum-Confined Stark Effect in Layered Hybrid Perovskites Mediated by Orientational Polarizability of Confined Dipoles. Nat. Commun. 2018, 9 , 4214.

(61) Wang, L.; Sasaki, T. Titanium Oxide Nanosheets: Graphene Analogues with Versatile Functionalities. Chem. Rev. 2014, 114, 9455-9486.

(62) Sasaki, T.; Watanabe, M. Osmotic Swelling to Exfoliation. Exceptionally High Degrees of Hydration of a Layered Titanate. J. Am. Chem. Soc. 1998, 120, 4682-4689.

(63) Takimoto, D.; Fukuda, K.; Miyasaka, S.; Ishida, T.; Ayato, Y.; Mochizuki, D.; Shimizu, W.; Sugimoto, W. 
Synthesis and Oxygen Electrocatalysis of Iridium Oxide Nanosheets. Electrocatalysis 2017, 8, 144150.

(64) Etman, A. S.; Asfaw, H. D.; Yuan, N.; Li, J.; Zhou, Z.; Peng, F.; Persson, I.; Zou, X.; Gustafsson, T.; Edström, K.; Sun, J. A One-Step Water Based Strategy for Synthesizing Hydrated Vanadium Pentoxide Nanosheets from $\mathrm{VO}_{2}(\mathrm{~B})$ as Free-Standing Electrodes for Lithium Battery Applications. J. Mater. Chem. A 2016, 4, 17988-18001.

(65) Liu, Z.; Ma, R.; Osada, M.; lyi, N.; Ebina, Y.; Takada, K.; Sasaki, T. Synthesis, Anion Exchange, and Delamination of Co-Al Layered Double Hydroxide: Assembly of the Exfoliated Nanosheet/Polyanion Composite Films and Magneto-Optical Studies. J. Am. Chem. Soc. 2006, 128, 4872-4880.

(66) Zhang, J.; Zhang, F.; Ren, L.; Evans, D. G.; Duan, X. Synthesis of Layered Double Hydroxide Anionic Clays Intercalated by Carboxylate Anions. Mater. Chem. Phys. 2004, 85, 207-214.

(67) Romero, R. B.; Ferrarezi, M. M. F.; Leite, C. A. P.; Alves, R. M. V.; Gonçalves, M. do C. Influence of the Layered Silicate Type on the Structure, Morphology and Properties of Cellulose Acetate Nanocomposites. Cellulose 2013, 20, 675-686.

(68) Benchakar, M.; Loupias, L.; Garnero, C.; Bilyk, T.; Morais, C.; Canaff, C.; Guignard, N.; Morisset, S.; Pazniak, H.; Hurand, S.; Chartier, P.; Pacaud, J.; Mauchamp, V.; Barsoum, M. W.; Habrioux, A.; Célérier, S. One MAX Phase, Different MXenes: A Guideline to Understand the Crucial Role of Etching Conditions on $\mathrm{Ti}_{3} \mathrm{C}_{2} \mathrm{~T}_{\mathrm{x}}$ Surface Chemistry. Appl. Surf. Sci. 2020, 530, 147209.

(69) Alhabeb, M.; Maleski, K.; Anasori, B.; Lelyukh, P.; Clark, L.; Sin, S.; Gogotsi, Y. Guidelines for Synthesis and Processing of Two-Dimensional Titanium Carbide ( $\left.\mathrm{Ti}_{3} \mathrm{C}_{2} \mathrm{~T}_{x} \mathrm{MXene}\right)$. Chem. Mater. 2017, 29, 7633-7644.

(70) Momma, K.; Izumi, F. VESTA: A Three-Dimensional Visualization System for Electronic and Structural Analysis. J. Appl. Crystallogr. 2008, 41, 653-658. 